\title{
Effectiveness of tigecycline-based versus colistin- based therapy for treatment of pneumonia caused by multidrug-resistant Acinetobacter baumannii in a critical setting: a matched cohort analysis
}

Yu-Chung Chuang ${ }^{1,2}$, Chien-Yu Cheng ${ }^{3}$, Wang-Huei Sheng ${ }^{1 *}$, Hsin-Yun Sun ${ }^{1}$, Jann-Tay Wang ${ }^{1}$, Yee-Chun Chen ${ }^{1,4}$ and Shan-Chwen Chang ${ }^{1,4}$

\begin{abstract}
Background: Colistin and tigecycline have both been shown good in vitro activity among multi-drug resistant Acinetobacter baumannii (MDRAB). A comparative study of colistin versus tigecycline for MDRAB pneumonia is lacking.

Methods: The study enrolled adults with MDRAB pneumonia admitted to intensive care units at a referral medical center during 2009-2010. Since there were no standardized minimum inhibitory concentration (MIC) interpretation criteria of tigecycline against $A$. baumannii, MIC of tigecycline was not routinely tested at our hospital. During the study periods, MIC of colistin was not routinely tested also. We consider both colistin and tigecycline as definite treatments of MDRAB pneumonia. Patients who received tigecycline were selected as potential controls for those who had received colistin. We performed a propensity score analysis, by considering the criteria of age, gender, underlying diseases, and disease severity, in order to match and equalize potential prognostic factors and severity in the two groups.

Results: A total of 294 adults with MDRAB pneumonia were enrolled, including 119 who received colistin and 175 who received tigecycline. We matched 84 adults who received colistin with an equal number of controls who received tigecycline. The two well matched cohorts share similar characteristics: the propensity scores are colistin: 0.37 vs. tigecycline: 0.37 , $(P=.97)$; baseline creatinine (1.70 vs. $1.81, P=.50)$, and the APACHE II score (21.6 vs. 22.0, $P=.99)$. The tigecycline group has an excess mortality of $16.7 \%$ ( $60.7 \%$ vs. $44 \%, 95 \%$ confidence interval $0.9 \%-32.4 \%, P=.04)$. The excess mortality of tigecycline is significant only among those with MIC $>2 \mu \mathrm{g} / \mathrm{mL}(10 / 12 \mathrm{vs} .37 / 84, P=.01)$, but not for those with $\mathrm{MIC} \leqq 2 \mu \mathrm{g} / \mathrm{mL}(4 / 10$ vs. $37 / 84, P=.81)$.
\end{abstract}

Conclusions: Our data disfavors the use of tigecycline-based treatment in treating MDRAB pneumonia when tigecycline and colistin susceptibilities are unknown, since choosing tigecycline-based treatment might result in higher mortality. The excess mortality of tigecycline-based group may be related to higher MIC of tigecycline (> $2 \mu \mathrm{g} / \mathrm{mL}$ ). Choosing tigecycline empirically for treating MDRAB pneumonia in the critical setting should be cautious.

Keywords: Acinetobacter baumannii, Pneumonia, Colistin, Tigecycline, Mortality, Nephrotoxicity

\footnotetext{
* Correspondence: whsheng@ntu.edu.tw

'Department of Internal Medicine, National Taiwan University Hospital, No. 7 Chung-Shan South Road, Taipei 100, Taiwan

Full list of author information is available at the end of the article
} 


\section{Background}

Healthcare-associated infections caused by Acinetobacter baumannii are increasing among patients in intensive care units (ICU) [1,2]. These infections are associated with a high mortality and a prolonged length of hospital stay [2]. Pneumonia is the most common clinical syndrome [3]. However, due to the emerging multi-drug resistance among $A$. baumannii isolates, the treatment choice for multi-drug resistant A. baumannii (MDRAB) related infection is limited [4].

Colistin and tigecycline have been shown to have good in vitro activity against $A$. baumannii pneumonia isolates, even in carbapenem-resistant isolates $[5,6]$. In fact, there have been successful experiences at using colistin in treating MDRAB pneumonia $[7,8]$, and colistin is recommended as a treatment option for pneumonia caused by MDRAB [9]. However, poor pulmonary penetration [10] and renal toxicity are the major concerns $[8,11]$. Tigecycline also presents good in vitro activity against MDRAB isolates [6], and several studies have revealed acceptable clinical responses of tigecycline for MDRAB pneumonia $[6,12,13]$. Though there are no available minimum inhibitory concentration (MIC) interpretation breakpoints of tigecycline against A. baumannii according to the criteria of the Clinical and Laboratory Standards Institute (CLSI) or the European Committee on Antimicrobial Susceptibility Testing (EUCAST) [2], tigecycline have been suggested as an alternative drug of choice in treating MDRAB pneumonia [14]. In spite of all this, a comparative study of colistin versus tigecycline for MDRAB pneumonia is lacking $[6,8,15]$. The aim of the current study is to compares the effectiveness and the adverse effects of colistin-based versus tigecyclinebased therapy in treating MDRAB pneumonia.

\section{Methods}

\section{Study population}

This is a retrospective study conducted among patients admitted to the ICU at the National Taiwan University Hospital (NTUH). NTUH is a 2,200-bed teaching hospital located in northern Taiwan that provides both primary and tertiary medical care. This study was approved by NTUH IRB committee.

Patients with MDRAB pneumonia and treated with colistin or tigecycline were enrolled in this study from January 2009 through December 2010. MDRAB was defined as $A$. baumannii, which showed non-susceptibility to $\geq 1$ agent in $\geq 3$ antimicrobial categories [16]. Pneumonia was defined based on a prospective surveillance by the microbiology lab and infection controls at NTUH [17]. A patient with a pneumonia must have either a new onset of purulent sputum, a change in the character of sputum, rales or dullness to percussion from a physical examination of the chest, chest radiographic examinations indicating new or progressive infiltrates, consolidation, cavitation, or pleural effusion and MDRAB isolated from the blood, bronchoscopic bronchial brushing, lavage or suitable sputum (defined as $>25$ polymorphonuclear neutrophil and $<$ 10 squamous epithelial cells per low power field). We used qualitative culture through the study. Ventilator-associated pneumonia (VAP) is defined as pneumonia developed $48 \mathrm{~h}$ or longer after mechanical ventilation is given by means of endotracheal intubation or tracheostomy. Patients are further classified by ventilator status: early-onset VAP $(<5 \mathrm{~d}$ of ventilation) or late-onset VAP ( $\geq 5 \mathrm{~d}$ of ventilation).

NTUH restricts the prescription of colistin or tigecycline to infectious disease physicians. In the circumstance of MDRAB infection, the prescriptions are limited to culture proven MDRAB, which showed non-susceptibility to ampicillin/sulbactam and the following anti-pseudomonas antibiotics, included cephalosporins, extended-spectrum penicillins, carbapenems, aminoglycosides, and fluoroquinolones. Since there were no MIC interpretation criteria of tigecycline against A. baumannii, MIC of tigecycline was not routinely tested at our hospital. During the study periods, MIC of colistin was not routinely tested also. Therefore, we consider both colistin and tigecycline as definite treatments of MDRAB pneumonia [2]. The dose of colistin (Colimycin injection, TTY Biopharm Co., Ltd., Taipei, Taiwan) was colistin base activity (CBA) $2.5-5 \mathrm{mg} /$ $\mathrm{kg} /$ day in 2-3 divided doses (2 million units or $160 \mathrm{mg}$ colistin methanesulfonate equivalent to $66.8 \mathrm{mg} \mathrm{CBA}$ ). The dose of tigecycline was a $100 \mathrm{mg}$ loading, followed by $50 \mathrm{mg}$ every 12 hours.

This study excluded patients under the age of 18 years or with incomplete medical records. We also excluded patients with concomitant infection, such as methicillin resistant Staphylococcus aureus or Pseudomonas aeruginosa. When patients had multiple episodes of MDRAB pneumonia and were treated with colistin or tigecycline, only the first episode was included.

\section{Microbiological studies}

This study identified A. baumannii by biochemical methods [18] and determined antimicrobial susceptibility by the disk diffusion method for gentamicin, amikacin, ciprofloxacin, levofloxacin, cefepime, ceftazidime, ticarcillin/ clavulanate, meropenem, and ampicillin/sulbactam according to CLSI criteria [19]. We performed MICs of colistin by agar dilution methods according to CLSI criteria [19]. Lastly, MICs of tigecycline were determined by agar dilution methods (BBL, BD Diagnostic Systems, Sparks, MD), with isolates of MIC $\leqq 2 \mu \mathrm{g} / \mathrm{mL}$ were considered as being susceptible [20].

\section{Data collection}

We collected demographic data, underlying diseases, and the concomitant infections by a retrospective chart review, 
recorded regimens of antimicrobial combination, and evaluated the severity of pneumonia by the Acute Physiology and Chronic Health Evaluation (APACHE) II score [21]. The outcomes for evaluation are inhospital mortality and nephrotoxicity. In patients with normal renal function, nephrotoxicity was defined as a doubling of baseline serum creatinine compared with treatment initiation, or a reduction in the calculated creatinine clearance $(\mathrm{CLCr})$ of $\geq 50 \%$. In patients with a pre-existing renal dysfunction, nephrotoxicity was defined as an increase of $>50 \%$ of the baseline creatinine level compared with the value at treatment initiation, a decrease of $\geq 20 \%$ of calculated serum CLCr from the baseline, or a decline in renal function that required renal replacement therapy $[7,22]$. Combination therapy was defined as at least 3 days of concurrent use of the antimicrobial agent other than colistin or tigecycline.

\section{Statistical analysis}

We calculated the mean and standard deviation (SD) for continuous variables and percentages for categorical variables. We then compared the associations between the clinical presentations of colistin and tigecycline treatments using Student's $t$ test or Fisher's exact test. A twosided $p$-value $\leq .05$ was considered significant.

Since there may be significant baseline characteristic differences between the colistin and tigecycline groups, we used propensity score matching to balance the baseline predictors for colistin or tigecycline use and mortality [23]. Propensity score matched analysis provides quasiexperimental design, which compared the outcome between two similar groups [24]. We estimated the probability of patients receiving colistin rather than tigecycline treatment with a logistic regression model, using their baseline characteristics before enrollment as variables. The fitted model was established by a stepwise variable selection procedure, with the significance levels for stay set to 0.2 . Age and gender were forced to be considered in the final model. We conducted all matching on a one-to-one basis without replacement, set the caliper at 0.25 , and chose the size of the caliper to be 0.0603 .

For the matched cohorts, this study examined postmatch balancing by using the paired $t$ test or McNemar's chi-square test [24]. We then calculated the risk difference (RD) in outcome, which included mortality, and nephrotoxicity, between the colistin group and the tigecycline group, or the average treatment effect of the treated (ATT). The data were analyzed with Stata software, version 12 (StataCorp, College Station, Texas). We used $R$ 2.14.2 software ( $R$ Foundation for Statistical Computing, Vienna, Austria) with the Package Matching version 4.8-0 to conduct propensity score matching and ATT estimation.

\section{Results}

Patient characteristics

A total of 294 ICU-admitted patients, who were found to have MDRAB pneumonia and were treated with colistin or tigecycline, were enrolled during the study period. Among the patients, 119 received colistin and 175 received tigecycline. Table 1 presents the demographic characteristics and underlying diseases of the 294 patients with MDRAB pneumonia. There were no significant differences among the different treatment groups for age, APACHE II score, and duration of treatment. However, patients treated with tigecycline had significantly lesser underlying malignancies $(1.1 \%$ vs. $28.6 \%$, $P<.001)$. The most common combination regimen used was carbapenem combination (34/294, 11.6\%).

There were no significant differences of underlying chronic kidney diseases $(23.5 \%$ vs. $17.7 \%, P=.24)$ and baseline creatinine (1.66 vs. $1.81, P=.40$ ) before treatment between the colistin and tigecycline groups. However, patients treated with colistin suffered from a significantly higher rate of nephrotoxicity $(10.1 \%$ vs. $3.4 \%, P=.03)$. There was no significant mortality rate difference between the colistin and tigecycline groups $(49.6 \%$ vs. $58.9 \%$, $P=.12)$. There was no significant mortality difference between carbapenem with the colistin combination $(n=15)$ and colistin without carbapenem combination $(\mathrm{n}=104)$ ( $46.7 \%$ vs. $50.0 \%, P=.81$ ). There was also no significant mortality difference between carbapenem with the tigecycline combination $(\mathrm{n}=19)$ and tigecycline without carbapenem combination $(\mathrm{n}=156)(42.1 \%$ vs. $60.9 \%, P=.12)$.

\section{Propensity score matching analysis}

We performed a multiple variables logistic analysis to determine the significant independent variables associated with the choice of colistin rather than tigecycline (Table 2). The factors associated with using colistin were male, better baseline creatinine, insulin use, and underlying malignancy. The large number of differences among the independent variables in the colistin and tigecycline groups did not permit a definitive assessment of the effect on the outcome. Accordingly, we employed a propensity matching analysis to control for the effect of the numerous confounders.

We matched 84 pairs of patients. There were no suitable tigecycline treatment controls for 35 unmatched colistin treated cases. After matching, the two groups of patients showed no significant differences of potential prognostic factors and severity of illness between them (Table 3$)$. The propensity scores were colistin: 0.370 vs. tigecycline: $0.370,(P=.97)$; underlying malignancy $(2.4 \%$ vs. $2.4 \%, P=1.00)$; and insulin use ( $23.8 \%$ vs. $25 \%, P=.85)$. 163 cases had positive sputum cultures of $A$. baumannii, among them 118 sputum samples were from tracheobronchial aspirate. 36 cases had positive bronchoscopic 
Table 1 Clinical and demographic characteristics of patients with multidrug resistant Acinetobacter baumannii pneumonia treated with colistin or tigecycline

\begin{tabular}{|c|c|c|c|c|}
\hline Characteristics & Total $\mathrm{N}=\mathbf{2 9 4}$ & Colistin $\mathrm{N}=119$ & Tigecycline $\mathrm{N}=175$ & $p$-value \\
\hline Age & $63.8(18.5)$ & $63.7(19.5)$ & $63.8(17.9)$ & .95 \\
\hline Male & $198(67.3)$ & $86(72.3)$ & $112(64)$ & .16 \\
\hline APACHE II score & $22.5(9.1)$ & $22.8(9.3)$ & $22.3(8.9)$ & .68 \\
\hline Length of hospital stay before enrollment & $32.0(36.9)$ & $33.2(45.9)$ & $31.2(29.4)$ & .64 \\
\hline \multicolumn{5}{|l|}{ Underlying diseases } \\
\hline Cardiovascular disease & $47(16.0)$ & $17(14.3)$ & $30(17.1)$ & .63 \\
\hline Diabetes mellitus & $84(28.6)$ & $33(27.7)$ & $51(29.1)$ & .90 \\
\hline Insulin use & $63(21.4)$ & $28(23.5)$ & $35(20)$ & .47 \\
\hline Chronic kidney disease & $59(20.1)$ & $28(23.5)$ & $31(17.7)$ & .24 \\
\hline Liver cirrhosis & $13(4.4)$ & $4(3.4)$ & $9(5.1)$ & .57 \\
\hline Chronic pulmonary disease & $30(10.2)$ & $15(12.6)$ & $15(8.6)$ & .33 \\
\hline Malignancy & $36(12.2)$ & $34(28.6)$ & $2(1.1)$ & $<.001$ \\
\hline Autoimmune & $16(5.4)$ & $5(4.2)$ & $11(6.3)$ & .60 \\
\hline Operation history & $140(47.6)$ & $51(42.9)$ & $89(50.9)$ & .19 \\
\hline \multicolumn{5}{|l|}{ Combination therapy } \\
\hline Aminoglycoside & $4(1.4)$ & $1(0.8)$ & $3(1.7)$ & .65 \\
\hline Carbapenem & $34(11.6)$ & $15(12.6)$ & $19(10.9)$ & .65 \\
\hline Sulbactam & $10(3.4)$ & $2(1.7)$ & $8(4.6)$ & .21 \\
\hline Baseline creatinine (mg/dL) & $1.75(1.47)$ & $1.66(1.43)$ & $1.81(1.50)$ & .40 \\
\hline Duration of treatment & $13.7(12.5)$ & $14.6(13.7)$ & $13.1(11.6)$ & .31 \\
\hline Follow-up duration & $32.4(31.5)$ & $33.1(30.9)$ & $31.9(32.0)$ & .75 \\
\hline
\end{tabular}

APACHE II, acute physiology and chronic health evaluation II.

bronchial lavage culture. The mean time $( \pm$ SD) elapsed between Acinetobacter isolation and the beginning of antibiotics of tigecycline or colistin group is $1.9( \pm 1.8)$ and $2( \pm 1.5)$ days, respectively $(P=.72)$. There were three (one Chryseobacterium indologenes, one Escherichia coli, and one Enterobacter aerogenes) polymicrobial infections in tigecycline and four (one C. indologenes, one E. coli, one Enterobacter cloacae, and one Klebesiella pneumoniae) in colistin group $(P=.71)$. In patient

Table 2 Logistic model for prediction of colistin rather than tigecycline use of the 294 enrolled patients

\begin{tabular}{llll}
\hline Variable & $\begin{array}{l}\text { Odds } \\
\text { ratio }\end{array}$ & $\begin{array}{l}\text { 95\% confidence } \\
\text { interval }\end{array}$ & $\boldsymbol{p}$-value \\
\hline Age (year) & 0.99 & $0.98-1.01$ & .69 \\
Male & 1.83 & $1.02-3.31$ & .04 \\
$\begin{array}{l}\text { Baseline creatinine }(\mathrm{mg} / \mathrm{dL}) \\
\text { Chronic kidney }\end{array}$ & 0.76 & $0.57-1.01$ & .06 \\
disease $\times$ Baseline creatinine & 1.42 & $1.09-1.84$ & .01 \\
$\begin{array}{l}\text { Insulin use } \\
\text { Malignancy }\end{array}$ & 1.55 & $0.82-2.93$ & .18 \\
\hline
\end{tabular}

Estimated area under the ROC curve $=0.74$. without renal dysfunction, the mean dose of colistin used was CBA $3.0( \pm 0.8) \mathrm{mg} / \mathrm{Kg} /$ day. All the distributions of the estimated propensity scores were wellbalanced after matching (Figure 1). We saw a significant mortality difference between the colistin and tigecycline groups $(44.1 \%$ vs. $60.7 \%)$, and the tigecycline group had a significant excess mortality (RD 16.7\%, 95\% confidence interval (C.I.) $0.9 \%-32.4 \%, P=.04$ ). The excess mortality remained significant even after being adjusted by propensity score, age, gender, and a combination of carbapenem use (adjusted RD 16.4\%, 95\% C.I. 0.9\% $31.8 \%, P=.04$ ) (Table 4 ). The colistin group had a higher rate of nephrotoxicity $(9.5 \%$ vs. $2.4 \%, P=.05)$. The excess nephrotoxicity remained significant even after being adjusted by propensity score (adjusted RD 7.4\%, $95 \%$ C.I. $1.2 \%-13.6 \%, \mathrm{P}=.02$ ).

The $\mathrm{MIC}_{50}$ and $\mathrm{MIC}_{90}$ of colistin were 1 and $2 \mu \mathrm{g} / \mathrm{mL}$, respectively. All tested isolates were susceptible to colistin. Among patients who were treated with tigecycline, only 22 have isolates available for tigecycline MIC testing. The $\mathrm{MIC}_{50}$ and $\mathrm{MIC}_{90}$ of tigecycline were 2 and $8 \mu \mathrm{g} / \mathrm{mL}$, respectively. The post-hoc analysis analyzing the mortality showed that the excess mortality of tigecycline is significant among those with MIC $>2 \mathrm{mg} / \mathrm{mL}(10 / 12$ vs. $37 / 84$, 
Table 3 Clinical and demographic characteristics of the matched patients with multidrug resistant Acinetobacter baumannii pneumonia treated with colistin or tigecycline

\begin{tabular}{|c|c|c|c|}
\hline Characteristics & $\begin{array}{l}\text { Colistin } \\
\mathrm{N}=84\end{array}$ & $\begin{array}{l}\text { Tigecycline } \\
\mathrm{N}=84\end{array}$ & $p$-value \\
\hline Age & $63.8(19.8)$ & $63.5(17.7)$ & .92 \\
\hline Male & $63(75)$ & $65(77.4)$ & .67 \\
\hline APACHE II score & $21.6(9.4)$ & $22.0(8.8)$ & .99 \\
\hline Ventilator-associated pneumonia & $58(69.1)$ & $60(71.4)$ & .74 \\
\hline Early onset VAP & $6(10.3)$ & $4(6.7)$ & .38 \\
\hline Late onset VAP & $52(90.0)$ & $56(93.3)$ & \\
\hline $\begin{array}{l}\text { Length of hospital stay before } \\
\text { enrollment }\end{array}$ & $29.5(33.7)$ & $29.0(30.1)$ & .92 \\
\hline $\begin{array}{l}\text { Length of ICU stay before } \\
\text { enrollment }\end{array}$ & $20.2(29.6)$ & $18.5(17.9)$ & .56 \\
\hline Duration of treatment & $14.0(11.2)$ & $12.9(9.3)$ & .51 \\
\hline Cardiovascular disease & $15(17.9)$ & $15(17.9)$ & 1.00 \\
\hline Diabetes mellitus & $24(28.6)$ & $27(32.1)$ & .59 \\
\hline Insulin use & $20(23.8)$ & $21(25)$ & .85 \\
\hline Chronic kidney disease & $22(26.2)$ & $22(26.2)$ & 1.00 \\
\hline Liver cirrhosis & $2(2.4)$ & $4(4.8)$ & .41 \\
\hline Chronic pulmonary disease & $8(9.5)$ & $8(9.5)$ & 1.00 \\
\hline Malignancy & $2(2.4)$ & $2(2.4)$ & 1.00 \\
\hline Autoimmune & $3(3.6)$ & $5(6.0)$ & .48 \\
\hline Operation history & $40(47.6)$ & $45(53.6)$ & .44 \\
\hline \multicolumn{4}{|l|}{ Combination therapy } \\
\hline Aminoglycoside & $1(1.2)$ & $2(2.4)$ & .56 \\
\hline Carbapenem & $9(10.7)$ & $9(10.7)$ & 1.00 \\
\hline Sulbactam & $1(1.2)$ & $3(3.6)$ & .32 \\
\hline Baseline creatine (mg/dL) & $1.70(1.40)$ & $1.81(1.60)$ & .50 \\
\hline Propensity score & $0.370(0.130)$ & $0.370(0.131)$ & .97 \\
\hline
\end{tabular}

APACHE II, acute physiology and chronic health evaluation II; ICU, intensive care units; VAP, ventilator-associated pneumonia.

$\mathrm{P}=.01)$, but not for those with $\mathrm{MIC} \leqq 2 \mathrm{mg} / \mathrm{mL}$ (4/10 vs. $37 / 84, \mathrm{P}=.81$ ).

We rechecked the result of the propensity score matching by a different matching scenario - the overfitted model - which means all baseline factors before colistin or tigecycline use were considered in the logistic regression model without stepwise selection. The results were similar. There was excess mortality of tigecycline (RD 19.7\%, $95 \%$ C.I. $2.8 \%-36.6 \%, P=.02$ ), and the colistin group had a significantly higher nephrotoxicity (RD 9.8\%, 95\% C.I. $1.1 \%-18.6 \%, P=.03)$.

\section{Discussion}

We found a significantly higher mortality rate in the tigecycline-based group and a higher rate of nephrotoxicity in the colistin-based group among the well-matched (a)

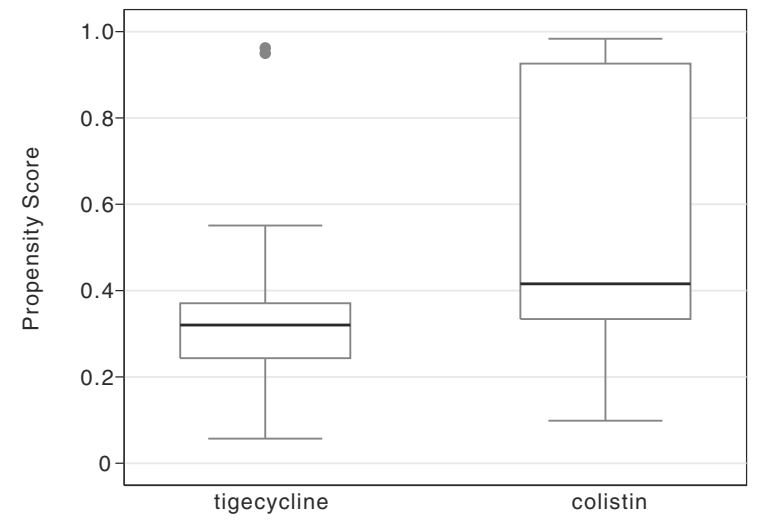

(b)

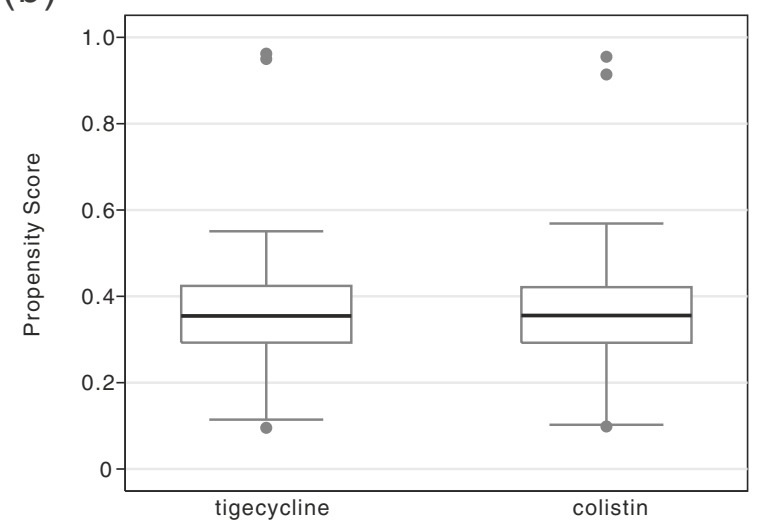

Figure 1 Boxplot comparing the distribution of the logit model of the estimated propensity scores (a) before and (b) after matching patients receiving colistin with those receiving tigecycline.

cohort. Post-hoc analysis showed that the mortality difference was noted in those with higher tigecycline MIC $(>2 \mu \mathrm{g} / \mathrm{mL})$.

There are concerns over tissue binding of colistin, poor colistin concentration in the lungs [10], and conversely tigecycline is concentrated in tissues, including lung parenchyma [25]. However, there were several meta-analysis showed excess overall mortality of tigecycline in the pooled analysis of different infection [26-28]. The higher mortality might be due to increased superinfections and more adverse events in the tigecycline groups [28], and an increased risk of death especially among patients with VAP [29]. The other reason for a significant treatment outcome difference may be partly due to colistin exhibiting even better in vitro activity to MDRAB [5]. In a previous study, Anthony et al. suggested that tigecycline MIC values in A. baumannii isolates may predict a clinical outcome [13]. Since our study is a retrospective design, not all isolates had post-hoc MIC testing. However, among the 104 preserved MDRAB isolates, the colistin susceptibility rate was $100 \%$, whereas 


\begin{tabular}{|c|c|c|}
\hline Variable & $\begin{array}{l}\text { Adjusted risk } \\
\text { differences \% }[95 \% \mathrm{Cl}]\end{array}$ & $p$-value \\
\hline Colistin vs. tigecycline & $-16.4[-0.9$ to -31.8$]$ & .04 \\
\hline Propensity score & $-30.3[-85.0$ to -24.3$]$ & .28 \\
\hline Age & $0.3[-0.07$ to -0.6$]$ & .11 \\
\hline Male & $-11.8[-29.5$ to -5.9$]$ & .19 \\
\hline Carbapenem combination & $-20.8[-45.8$ to -4.2$]$ & .10 \\
\hline
\end{tabular}

the tigecycline susceptibility rate was only $50.0 \%$. Though tigecycline is concentrated in tissues, including lung parenchyma, the concentration in epithelial lining fluid is relatively low, with Cmax only $0.37 \mu \mathrm{g} / \mathrm{mL}$, especially compared to high MICs of tigecycline to A. baumannii [25]. Though, while a high dose of tigecycline achieves a higher Cmax, and might have better clinical efficacy [30], high dose of tigecycline was not practiced at NTUH. Recent study revealed that previous colistin dosage used might be under dosing, which may possibly underestimate the effectiveness of colistin in our cohort [31].

Combination therapy for MDRAB infection is controversial. The combination of tigecycline with carbapenem showed a comparable outcome than tigecycline monotherapy [12], while the combination of colistin with carbapenem showed a less favorable outcome than colistin monotherapy [32]. According to Taiwanese regulations (Regulations for National Health Insurance Reimbursement), only few cases in out cohort had received tigecycline or colistin combination therapy. Although our study did not support the effectiveness of combination therapy for MDRAB pneumonia, further large sample sized prospective studies are warrant to illustrate the issue.

Colistin presented nephrotoxicity in around $6 \%$ of treated patients [8]. However, in the meta-analysis of the two arms study, Florescu et al. reported no significant higher rates of nephrotoxicity in the colistin group than that in the comparator group (Odds ratio 1.14, 95\% C.I. $0.59-2.20$, $P=.69$ ). The difference may be due to the fact that the baseline characteristics of the colistin and comparator groups might be different especially in the observational studies, but they were not adjusted in the meta-analysis. In our study we showed borderline significant excess nephrotoxicity of $7.1 \%$ of the colistin group compared to tigecycline group $(P=.05)$ for the baseline characteristic comparable cohorts. Thus, for patients with predisposing factors such as nephrotoxic drugs, chronic kidney diseases, and hypovolemia, we should carefully monitor the renal function during colistin use [11].

This study has several limitations. First, the majority of the patients were diagnosed using qualitative sputum culture, which might result in over-diagnosis of pneumonia. Hence, the difference of the effectiveness between tigecycline and colistin might be underestimated. Second, there are difficulties inherent in the design of any retrospective matched cohort study. We may have missed some unobserved confounders that were unbalanced, even though the fitness of our prediction models appear to be good (area under ROC: 0.74), and the result is robust between different matching scenarios. In order to avoid any possible misclassification bias in the retrospective design, a hard end point - mortality - is used rather than clinical improvement. Third, since there were no MIC interpretation criteria of tigecycline against A. baumannii [2], our microbiology lab had no routine antimicrobial susceptibility test of tigecycline. Thus not all isolates had tigecycline susceptibility tested. Lastly, in our lab, the MIC testing of colistin and tigecycline were uniformly performed using agar dilution. The result of broth microdilution might be affected by whether fresh medium was used or Oxyrase was added, but agar dilution might not $[33,34]$. However, limited study showed that the MIC obtained by agar dilution is lower than that obtained by broth dilution techniques [33].

\section{Conclusions}

In patients with possible nephrotoxic factors, their renal function during colistin use should be carefully monitored. Our data disfavors the use of tigecycline-based treatment in treating MDRAB pneumonia when tigecycline and colistin susceptibilities are unknown, since choosing tigecyclinebased treatment might result in higher mortality. The excess mortality of tigecycline-based group may be related to higher MIC of tigecycline $(>2 \mu \mathrm{g} / \mathrm{mL})$. There is an urgent need to establish the interpretation criteria of tigecycline for MDRAB infection. The effectiveness of tigecycline use in those with low tigecycline MIC in the era of emerging MDRAB warrants further study.

\section{Abbreviations}

APACHE: Acute Physiology and Chronic Health Evaluation; ATT: The average treatment effect of the treated; CBA: Colistin base activity; C.I.: Confidence interval; CLCr: Calculated creatinine clearance; CLSI: Clinical and Laboratory Standards Institute; EUCAST: European Committee on Antimicrobial

Susceptibility Testing; ICU: Intensive care units; MDRAB: Multi-drug resistant Acinetobacter baumannii; MIC: Minimum inhibitory concentration;

NTUH: National Taiwan University Hospital; RD: Risk difference; SD: Standard deviation.

\section{Competing interests}

The authors declare that they have no competing interests.

Authors' contributions

YCC, and CYC reviewed the medical records and drafted the manuscript; YCC, HYS, and WJT analyzed and interpreted the data. WHS designed and oversaw the study, analyzed and interpreted the data, and revised the manuscript. YCC and SCC revised the manuscript. All authors have read and approved the manuscript for publication. 


\section{Acknowledgments}

This study was supported partially by the National Science Council, Taiwan (NSC-101-2314-B-002-089-MY3 and NSC-101-2314-B-002-135-MY3) and by Department of Health Taiwan (DOH100-TD-B-111-001).

No funding sources had any role in the design or conduct of the study; collection, management, analysis, or interpretation of the data; or preparation, review, or approval of the manuscript.

Preliminary analyses of these data were presented as abstract no. K184 at the 53th Interscience Conference on Antimicrobial Agents and Chemotherapy, Denver, 10-13 September, 2013.

\section{Author details}

${ }^{1}$ Department of Internal Medicine, National Taiwan University Hospital, No. 7 Chung-Shan South Road, Taipei 100, Taiwan. ${ }^{2}$ Graduate Institute of Clinical Medicine, College of Medicine, National Taiwan University, Taipei, Taiwan. ${ }^{3}$ Department of Internal Medicine, Tao-Yuan General Hospital, Tao-Yuan, Taiwan. ${ }^{4}$ Department of Medicine, National Taiwan University College of Medicine, Taipei, Taiwan.

Received: 28 October 2013 Accepted: 10 February 2014

Published: 24 February 2014

\section{References}

1. Garcia-Garmendia JL, Ortiz-Leyba C, Garnacho-Montero J, Jimenez-Jimenez FJ, Perez-Paredes C, Barrero-Almodovar AE, Gili-Miner M: Risk factors for Acinetobacter baumannii nosocomial bacteremia in critically ill patients: a cohort study. Clin Infect Dis 2001, 33(7):939-946.

2. Peleg AY, Seifert $H$, Paterson DL: Acinetobacter baumannii: emergence of a successful pathogen. Clin Microbiol Rev 2008, 21(3):538-582.

3. Karageorgopoulos DE, Falagas ME: Current control and treatment of multidrug-resistant Acinetobacter baumannii infections. Lancet Infect Dis 2008, 8(12):751-762.

4. Fishbain J, Peleg AY: Treatment of Acinetobacter infections. Clin Infect Dis 2010, 51(1):79-84.

5. Dizbay M, Altuncekic A, Sezer BE, Ozdemir K, Arman D: Colistin and tigecycline susceptibility among multidrug-resistant Acinetobacter baumannii isolated from ventilator-associated pneumonia. Int $J$ Antimicrob Agents 2008, 32(1):29-32.

6. Karageorgopoulos DE, Kelesidis T, Kelesidis I, Falagas ME: Tigecycline for the treatment of multidrug-resistant (including carbapenem-resistant) Acinetobacter infections: a review of the scientific evidence. J Antimicrob Chemother 2008, 62(1):45-55.

7. Garnacho-Montero J, Ortiz-Leyba C, Jimenez-Jimenez FJ, Barrero-Almodovar AE, Garcia-Garmendia JL, Bernabeu-Wittel IM, Gallego-Lara SL, Madrazo-Osuna $\mathrm{J}$ : Treatment of multidrug-resistant Acinetobacter baumannii ventilator-associated pneumonia (VAP) with intravenous colistin: a comparison with imipenem-susceptible VAP. Clin Infect Dis 2003, 36(9):1111-1118.

8. Florescu DF, Qiu F, McCartan MA, Mindru C, Fey PD, Kalil AC: What is the efficacy and safety of colistin for the treatment of ventilator-associated pneumonia? a systematic review and meta-regression. Clin Infect Dis 2012, 54(5):670-680

9. American Thoracic Society; Infectious Diseases Society of America: Guidelines for the management of adults with hospital-acquired, ventilator associated, and healthcare-associated pneumonia. Am J Respir Crit Care Med 2005, 171(4):388-416.

10. Imberti R, Cusato M, Villani P, Carnevale L, lotti GA, Langer M, Regazzi M: Steady-state pharmacokinetics and BAL concentration of colistin in critically III patients after IV colistin methanesulfonate administration. Chest 2010, 138(6):1333-1339.

11. Spapen H, Jacobs R, Van Gorp V, Troubleyn J, Honore PM: Renal and neurological side effects of colistin in critically ill patients. Ann Intensive Care 2011, 1(1):14.

12. Schafer JJ, Goff DA, Stevenson KB, Mangino JE: Early experience with tigecycline for ventilator-associated pneumonia and bacteremia caused by multidrug-resistant Acinetobacter baumannii. Pharmacotherapy 2007, 27(7):980-987.

13. Anthony KB, Fishman NO, Linkin DR, Gasink LB, Edelstein PH, Lautenbach E: Clinical and microbiological outcomes of serious infections with multidrug-resistant gram-negative organisms treated with tigecycline. Clin Infect Dis 2008, 46(4):567-570
14. Jean SS, Hsueh PR: Current review of antimicrobial treatment of nosocomial pneumonia caused by multidrug-resistant pathogens. Expert Opin Pharmacother 2011, 12(14):2145-2148.

15. Ku K, Pogue JM, Moshos J, Bheemreddy S, Wang Y, Bhargava A, Campbell M, Khandker N, Lephart PR, Chopra T, Hayakawa K, Martin ET, AbreuLanfranco O, Dhar S, Kaye KS, Marchaim D: Retrospective evaluation of colistin versus tigecycline for the treatment of Acinetobacter baumannii and/or carbapenem-resistant Enterobacteriaceae infections. Am J Infect Control 2012, 40(10):983-987.

16. Magiorakos AP, Srinivasan A, Carey RB, Carmeli Y, Falagas ME, Giske CG, Harbarth S, Hindler JF, Kahlmeter G, Olsson-Liljequist B, Paterson DL, Rice LB, Stelling J, Struelens MJ, Vatopoulos A, Weber JT, Monnet DL: Multidrugresistant, extensively drug-resistant and pandrug-resistant bacteria: an international expert proposal for interim standard definitions for acquired resistance. Clin Microbiol Infect 2012, 18(3):268-281.

17. Chuang YC, Chen YC, Chang SC, Sun CC, Chang YY, Chen ML, Hsu LY, Wang JT: Secular trends of healthcare-associated infections at a teaching hospital in Taiwan, 1981-2007. J Hosp Infect 2010, 76:143-149.

18. Schreckenberger PC, von Graevenitz A: Acinetobacter, Achromobacter, Acaligenes, Moraxella, Methylobacterium, And Other Nonfermentative Gram-Negative Rods. In Manual Of Clinical Microbiology. 7th edition. Edited by Murray EJB PR, Pfaller MA, Tenover FC, Yolken RH. Washington, D.C: ASM Press; 2000:539-560.

19. Clinical and Laboratory Standards Institute: Performance Standards For Antimicrobial Susceptibility Testing: 17th Informational Supplement, M100-S17. Wayne, PA: CLSI; 2007.

20. Fernandez-Mazarrasa C, Mazarrasa O, Calvo J, del Arco A, Martinez-Martinez L: High concentrations of manganese in Mueller-Hinton agar increase MICs of tigecycline determined by Etest. J Clin Microbiol 2009, 47(3):827-829.

21. Napolitano LM: Use of severity scoring and stratification factors in clinical trials of hospital-acquired and ventilator-associated pneumonia. Clin Infect Dis 2010, 51(Suppl 1):S67-S80.

22. Cheng $C Y$, Sheng WH, Wang JT, Chen YC, Chang SC: Safety and efficacy of intravenous colistin (colistin methanesulphonate) for severe multidrugresistant Gram-negative bacterial infections. Int J Antimicrob Agents 2010, 35(3):297-300.

23. Rosenbaum PR, Rubin DB: The central role of the propensity score in observational studies for causal effects. Biometrika 1983, 70(1):41-55.

24. Luo Z, Gardiner JC, Bradley CJ: Applying propensity score methods in medical research: pitfalls and prospects. Med Care Res Rev 2010, 67(5):528-554

25. Conte JE Jr, Golden JA, Kelly MG, Zurlinden E: Steady-state serum and intrapulmonary pharmacokinetics and pharmacodynamics of tigecycline. Int J Antimicrob Agents 2005, 25(6):523-529.

26. Prasad P, Sun J, Danner RL, Natanson C: Excess deaths associated with tigecycline after approval based on noninferiority trials. Clin Infect Dis 2012, 54(12):1699-1709.

27. Cai Y, Wang R, Liang B, Bai N, Liu Y: Systematic review and meta-analysis of the effectiveness and safety of tigecycline for treatment of infectious disease. Antimicrob Agents Chemother 2011, 55(3):1162-1172.

28. Yahav D, Lador A, Paul M, Leibovici L: Efficacy and safety of tigecycline: a systematic review and meta-analysis. J Antimicrob Chemother 2011, 66(9):1963-1971.

29. Food and Drug Administration: FDA drug safety communication: increased risk of death with Tygacil (tigecycline) compared to other antibiotics used to treat similar infections. Available at: http://www.fda.gov/Drugs/DrugSafety/ ucm224370.htm. Accessed 17 February 2014

30. Ramirez J, Dartois N, Gandjini H, Yan JL, Korth-Bradley J, McGovern PC: Randomized phase 2 trial to evaluate the clinical efficacy of two high-dosage tigecycline regimens versus imipenem-cilastatin for treatment of hospital-acquired pneumonia. Antimicrob Agents Chemother 2013, 57(4):1756-1762.

31. Dalfino L, Puntillo F, Mosca A, Monno R, Spada ML, Coppolecchia S, Miragliotta G, Bruno F, Brienza N: High-dose, extended-interval colistin administration in critically ill patients: is this the right dosing strategy? a preliminary study. Clin Infect Dis 2012, 54(12):1720-1726.

32. Falagas ME, Rafailidis PI, Kasiakou SK, Hatzopoulou P, Michalopoulos A: Effectiveness and nephrotoxicity of colistin monotherapy vs. colistin-meropenem combination therapy for multidrug-resistant 
Gram-negative bacterial infections. Clin Microbiol Infect 2006,

12(12):1227-1230.

33. Hope R, Warner M, Mushtag S, Ward ME, Parsons T, Livermore DM: Effect of medium type, age and aeration on the MICs of tigecycline and classical tetracyclines. J Antimicrob Chemother 2005, 56(6):1042-1046.

34. Bradford PA, Petersen PJ, Young M, Jones $\mathrm{CH}$, Tischler M, O'Connell J: Tigecycline MIC testing by broth dilution requires use of fresh medium or addition of the biocatalytic oxygen-reducing reagent oxyrase to standardize the test method. Antimicrob Agents Chemother 2005, 49(9):3903-3909.

doi:10.1186/1471-2334-14-102

Cite this article as: Chuang et al:: Effectiveness of tigecycline-based versus colistin- based therapy for treatment of pneumonia caused by multidrug-resistant Acinetobacter baumannii in a critical setting: a matched cohort analysis. BMC Infectious Diseases 2014 14:102.

\section{Submit your next manuscript to BioMed Central and take full advantage of:}

- Convenient online submission

- Thorough peer review

- No space constraints or color figure charges

- Immediate publication on acceptance

- Inclusion in PubMed, CAS, Scopus and Google Scholar

- Research which is freely available for redistribution 